\title{
How Plesiosaurs Swam: New Insights into Their Underwater Flight Using "Ava", a Virtual Pliosaur
}

\author{
Max Hawthorne ${ }^{1, *}$, Mark A. S. McMenamin ${ }^{2}$, Paul de la Salle ${ }^{3}$ \\ ${ }^{1}$ Far From The Tree Press, LLC, 4657 York Rd., \#952, Buckingham, PA, 18912, United States \\ ${ }^{2}$ Department of Geology and Geography, Mount Holyoke College, South Hadley, Massachusetts, United States \\ ${ }^{3}$ Swindon, England \\ *Correspondence: author@maxhawthorne.com; Tel.: 267-337-7545
}

\begin{abstract}
Analysis of plesiosaur swim dynamics by means of a digital 3D armature (wireframe "skeleton") of a pliosauromorph (“Ava") demonstrates that: 1, plesiosaurs used all four flippers for primary propulsion; 2 , plesiosaurs utilized all four flippers simultaneously; 3 , respective pairs of flippers of Plesiosauridae, front and rear, traveled through distinctive, separate planes of motion, and; 4 , the ability to utilize all four paddles simultaneously allowed these largely predatory marine reptiles to achieve a significant increase in acceleration and speed, which, in turn, contributed to their sustained dominance during the Mesozoic.
\end{abstract}

Keywords aquatic reptiles, plesiosaurs, pliosaurs, swim kinematics, Strouhal numbers

\section{Introduction}

Plesiosauridae are a clade of extinct Mesozoic marine reptiles with a long evolutionary history. Since their discovery in 1821, the precise method of their swim locomotion has remained unsolved, although many hypotheses have been proposed [1-6]. Previous studies have posited a variety of locomotory techniques to offset the implied redundancy of the paired paddles working in conjunction while moving through the same subaqueous plane; i.e. a duplication of resistance that generated little or no additional thrust (herein referred to as the "principal of flipper redundancy"). The only thing certain is that plesiosaurs had abandoned the tail propulsion common in earlier aquatic reptiles [7]. Mosasaurs and ichthyosaurs, in contrast, continued with tail propulsion until the Cretaceous [8]

Modelers have proposed front only (or front primary) means of propulsion, a rear only (or rear primary) means of propulsion, an alternate paddle application means of propulsion, and a figure-eight pattern paddle application [9].
Further study attempted to justify the use of all four flippers simultaneously via the use of paddle-generated vortices, which require specific timing to achieve optimal additional thrust. These attempts have largely relied on anatomical studies of strata-compressed plesiosaur skeletons, and/or preconceived notions as pertains to the paddles' inherent ranges of motion [8, 10-12]. What has not been considered are the opposing angles of the pectoral and pelvic girdles, which strongly indicate varied-yet-complementing relations between the front and rear sets of paddles, both in repose and in motion, and imply separate planes of motion for both.

The main argument when it comes to defining plesiosaur locomotion has been centered on the notion that two sets of flippers cannot be effectively deployed simultaneously for an effective thrust stroke. If they were, they would be pushing the same water, hence the animal couldn't go significantly faster. As per the principle of flipper redundancy, why use four fins to do a job when two are sufficient, as in sea lions? Many hypotheses have been put forward to explain why plesiosaurs had four flippers and also to explain how they used them. These included: the use of alternate strokes (front then rear) which enabled one set of flippers to rest while the other worked; the use of rear or front flippers as a primary means of propulsion (with the remaining set delegated mainly to steering), and; the "vortices theory", where paddle-generated vortices assist the animal's recovery stroke. Do any of these previous hypotheses explain plesiosaur locomotion? If not, how did the four paddles function?

Based on an overview of known species, we believe the concept of an alternate flipper or figure-eight pattern is mistaken. If we consider modern marine life: fish, cetaceans, or the usual list of tetrapods compared to plesiosaurs, due to their reliance on a similar, albeit single set of flippers for propulsion (penguins, pinnipeds and sea turtles), we see that a single caudal fin or set of paddles is sufficient. There is no advantage for a duplicate or redundant set of flippers, i.e. no extant species uses two sets of equally-developed flippers with one pair that must "rest" during its negative stroke in 
order to recover. Therefore, there must be a locomotory advantage for the development of four, relatively equal paddles.

Based on available evidence, we believe that the notion of a front or rear-only swim pattern is even less plausible. When examining the skeletons of plesiosaurs, we posit here a unique radial adaptation of the shoulder and a similar mechanism developed in the pelvis. The bones for both pairs of flippers are extremely well-developed with large muscle attachment points (Figure 1). The muscles powering these paddles were undoubtedly huge and made for strength and endurance.

Modern examples provide some context for our hypothesis. Consider the recently discovered waterfall-climbing blind cave fish, Cryptotora thamicola [13]. It has developed a pelvis and a stiffened spine, giving it the ability to utilize four comparably developed fins for locomotion. It uses all of them simultaneously, with a tetrapod-like gait that resembles that of a salamander. Consider also the case of extant sea turtles (Fig. 2). The large, well-developed forelimbs are the propulsion units and are also responsible for major directional changes, whereas the smaller, more restricted rear flippers function mainly as rudders to keep the animal on an even keel, assist in turning, and, in the case of females, for nest excavation. Two swimming gaits have been characterized in turtles [14]. A similar configuration of smaller rear flippers was employed by the parvipelvian ichthyosaurs. Small flippers are quite adequate for steering purposes.

The hypothesis that a plesiosaur's rear flippers benefited from dual lines of vortices (referred to as the "vortex street") generated by the front set depends on both sets of paddles moving through the same planes of motion, as well as at a certain pace and spacing, in order to effectively generate additional thrust [6]. Both marine and terrestrial tetrapods rely on a varying gait based on circumstances (changing direction, accelerating, etc.), and would be ill-equipped to survive were they limited to a unique, "optimal" speed/phasing in order to derive maximum benefits. The robot used to test the hypothesis [6] was equipped with a paddle that allows rotational freedom of movement.

We propose that skeletal adaptations in plesiosaurs enabled them to utilize all four flippers simultaneously, and in such a way that the paddles not only avoided violating the principal of flipper redundancy but also complemented one another by moving in conjunction through distinctly separate planes of motion. By doing so, this adaptation enabled the animal to generate significantly more thrust, increasing both its ability to accelerate as well as its maximum speed.

Range of motion limitations indicated by studying non-compressed skeletons of plesiosaurs suggest that the front flippers pushed down and back, in a pattern similar to that of a penguin, while the rear flippers pushed more horizontally and backwards. The front flippers therefore moved through a lower plane of motion than the rear, thus pushing water in a different range. When working in combination they moved twice as much water, giving the animal distinctive and unique advantages, comparable to what one might see in a fish or whale with a tail twice the original size or a sea lion with two sets of flippers.

\section{Methodology}

For purposes of this study, a multi-pronged approach was adopted. This included comparison studies of physical and photographic evidence of the skeletal remains of an assortment of known Plesiosauridae forms, including plesiosauromorph and pliosauromorph body types, as well as polycotylids. Comparative references were also made with extant tetrapods (Cheloniidae and Spheniscidae) which utilize front-paddle-only, dual-paddle locomotory methods similar to plesiosaurs. Data acquired from the aforementioned anatomical studies was utilized in the development of a computer model which, taking water resistance into account, went through various iterations until it successfully recreated a functional plesiosaur swim cycle, one that mimics the underwater movements of these remarkable marine Sauropsida.

As previously stated, traditional views and the subsequent positioning of plesiosaur paddles in attempted motion reconstructions have been largely based on taphonomically flattened remains and/or the assumption that the flippers moved in a restricted range of motion (Fig. 3). This applies to almost all previously published theories [6, 15]. However, when observing a more scientifically accurate, non-compressed skeleton (Figs. 3-4), it becomes obvious that there are inherent differences in the respective ranges of motion of the front and rear paddles, as evidenced by morphological adaptations of the skeleton, in particular, at the humeral and femoral insertions. The elongate, blade-like dorsal process of the clavicle of the polycotylid plesiosaur Manemergus anguirostris from the Turonian of Morocco [16], for example, indicates that the third dimension is critial for understanding muscle attachments and range-of-motion.

When observing the fore-flippers' humeral insertion point (Figure 4), it is evident that the pectoral/shoulder girdle angles upward, toward the animal's cranium. This elevation of the shoulder girdle implies that, when generating thrust, the front paddles were able to angle upward and sweep further forward on the negative stroke that the rear set, coming back and down powerfully on the positive. The power-stroke from the front limbs/paddles traveled through a largely vertical movement, not unlike that of a penguin, except with a bit more range and flexibility, and most likely terminated in the same direction shown in Figure 5.

When observing the femoral insertion point where the rear flippers insert into the pelvic girdle, we find that the pelvis is angled in the reverse - up toward the caudal vertebrae (Figure 4). This change in angle/range of motion physically 
restricted the animal's ability to thrust down hard during a running speed downstroke, but, at the same time, it enabled it to raise its rear set of flippers higher in the water throughout its stroke, pushing backward and ending in a far more lateral termination point than the front set. This served the purpose of encompassing a largely different plane of motion, enabling the plesiosaur to thrust back hard with its posterior paddles, most likely finishing in a position more aligned with its body (Figure 6).

\section{Strouhal Number at Cruising Speed}

The main goal of our digital animation model, Ava (a generalized brachauchenine thalassophonean pliosaurid close to Kronosaurus boyacensis), is to assess the swimming mechanics of an ancient marine predator. Before proceeding to the results of the simulation, we report here a simple goodness-of-fit test for the model to determine if it generates plausible metrics in terms of cruising speed swim using some reasonable assumptions.

This involves a calculation of a dimensionless number, the Strouhal value for Ava's cruising speed. Strouhal number (St), a measure of the efficiency of propulsion, is calculated as:

$$
\mathrm{St}=\mathrm{fA} / \mathrm{U}
$$

where f equals stroke frequency, A equals stroke amplitude, and $U$ equals forward speed. Assuming a pliosaur $11 \mathrm{~m}$ length, we estimate a cruising speed stroke frequency (f) of $0.5 \mathrm{beats} / \mathrm{sec}$, a stroke amplitude of $1.48 \mathrm{~m}$, and a cruising speed of 3 mps.

A frequency of one stroke every two seconds is identical to that of a cruising leatherback (Dermochelys corinacea). Using the above values gives Ava's Strouhal number as:

$$
\mathrm{St}_{\mathrm{A}}=0.247 \text {. }
$$

Strouhal number peaks in living aquatic tetrapod lineages at between $0.2<\mathrm{St}<0.4$. This has been considered a case of tuning for higher power efficiency [17]. At approximately $2.5, \mathrm{St}_{\mathrm{A}}$ is well within this range, showing that the Ava model generates a reasonable Strouhal value when calculated with reasonable stroke frequency and speed assumptions. Stroke amplitude was directly measured from a still shot of the animation, and was measured without consultation with or knowledge of our animator Mathieu Lafreniere, and so was largely independent of rendering the animation itself.

$\mathrm{St}_{\mathrm{A}}$ is in the lower half of the Strouhal peak efficiency zone, but this might not be unexpected for a predatory animal that might be expected to have optimized its burst or running speed at the expense of its cruising speed. We do not attempt here to calculate Strouhal value for burst speed, as burst speed estimates are poorly constrained at this time (see below).

\section{Comparative Swim Kinematics}

Evolutionary adaptations to the rib cages of plesiosaurs support our hypothesis, that is, simultaneous use of all four flippers. Both the pectoral and sacral ribs observed in many plesiosaurs are characteristically shortened, the sacral ribs above the pelvic girdle typically more so than those above the pectoral (Figures 4, 7). As in sea turtles, which often have an "alcove" in their shells to accommodate their front flippers upward lift (Figure 8), morphological adaptations typically reduce friction and impacts by moving body parts.

Turtle flippers have large, powerful muscles, and as they are flexing and hoisting the flippers up and back both those muscles, and the flippers themselves, would rub against ribs that extended too far down. Hence, they became reduced in length. In plesiosaurs, the amount of adaptive rib shortening that occurs is consistent with our theorized range of motion for the respective flipper pairs. We see standard, esophageal-accommodating shortening in the cervical ribs, moderate, but more pronounced, pectoral rib shortening for the front set of paddles and extreme shortening, combined with upward angling, of the sacral ribs, to accommodate both the higher range of motion of the rear paddles and the flexion of the muscles driving them (Figures 7, 9).

This pattern of adaptive rib shortening occurs repeatedly in assorted plesiosaur species, with homoplasy occurring in the angles of the front and rear limb girdles, with the greatest degree of shortening taking place in the pelvic/sacral region (Figures 7-9). This allowed the animal's front limbs to move in a pattern somewhat akin to a penguin or sea turtle (sans the chelonian's full upper range, due to the absence of an elbow joint), with the rear flippers taking advantage of their ability to reach higher and encompassing water the front flippers were unable catch. During the return, reverse, or negative stroke, the movement of the two sets would have been similar. It is likely that, during high speed pursuits, the rear flippers benefited from the suction/vacuum generated by the front flippers during their reverse stroke. The rear pair could practically come along for the ride, conserving their strength and absorbing kinetic energy to allow for a more powerful positive stroke.

This pattern of movement is supported by Frank Sanders' and Kenneth Carpenter's 2010 study [18], which concluded that the flipper movement in plesiosaurs was largely vertical: "The best modern anatomical analogs are sea turtles and underwater birds, particularly penguins. All of these animals swim with variations of underwater flight. In particular, they all move their limbs mainly vertically, with some fore-to-aft 
motion and slight axial rotation, all of which is possible for the plesiosaur limbs."

Consideration of this largely vertical movement, but taking into account the adjusted ranges of motion (implied by the opposingly angled pectoral and pelvic girdles of plesiosaurs), demonstrates how these animals were able to utilize both sets of paddles - front and rear - moving them through separate planes or motion, and to maximum benefit.

Using the above joint motion constraints and the resultant theoretical ranges of motion as a baseline, and taking into account the separate planes of motion for both the front and rear paddles, a digital 3D armature (wireframe "skeleton") of a pliosauromorph (known as "Ava") was then constructed using the Autodesk Maya 3D Computer Graphics Application. Assorted ranges of motion and combinations of flipper movements and angles were utilized in the experiment, including the incorporation of various rhythms and speeds.

Once the "flesh" was added to Ava's armature, we studied footage of extant penguins, sea lions, and sea turtles to help us understand and take into account directional changes that took place based on the angles of each thrust, as well as the effects said thrust had on the animal's body. Issues of stability were addressed, with the final model/sequence goal to develop a virtual plesiosaur that could perform a perfected swim cycle at various speeds, including rapid acceleration and gliding.

\section{Results and Discussion}

As the accompanying video demonstrates, the results of our study indicated that plesiosaurs did indeed utilize both sets of paddles for locomotion. Moreover, they used them simultaneously, with the flippers traveling through separate planes of motion to not only maximize benefits in terms of generating initial thrust, but also to achieve higher top end speed as well as increased maneuverability. Over the course of our study our virtual pliosaur Ava went through numerous revisions. Each iteration took into account studies of the skeletal remains of assorted plesiosaur species (including diagrams, photographs, and videos), as well as extant marine tetrapods. These included observations/motion studies of extant marine tetrapods (and any anatomical constraints imposed by same).

\section{Eliminating sea lion and rowing models}

Our findings concurred at least in part with a previous study, which stated both the "sea lion" and "rowing" models $[2,19,20]$ for plesiosaurs are contraindicated. As stated by reference 5: "Our analysis demonstrates that the sea lion- and rowing- models [for plesiosaurs] are kinematically impossible due to the prominent glenoid processes that would restrict the necessary posterior motion. Anatomical comparisons with extant tetrapod skeletons show that the glenohumeral joint of plesiosaurs is closest to that of underwater fliers such as sea turtles and penguins."

As can be observed in this still frame (dorsal view) from one of Ava's wireframe test runs (Figure 10), the posterior range of motion of the paddles, particularly the rear set, far exceeds feasible/safe ranges of motion for the animal and, if enacted, would have resulted in the paddle being partially, if not completely, dislocated at the femoral insertion point. A far more likely posterior range of motion for the rear paddles is indicated in red. This posterior range of motion is in line with previously published estimates for maximum anteroposterior ranges of motion for the rear paddles, i.e. approximately -57 degrees [21]. A more anatomically correct posterior range for the paddles (the posterior set in particular) can be seen in the wireframe still of Ava (Figure 11).

\section{Increased posterior range of motion for the rear flippers}

As previously stated, when observing both the caudal-oriented up-angling of the plesiosaur pelvis, as well as the adaptive shortening of the sacral ribs to accommodate the accompanying muscles, we calculated that the range of motion for the posterior paddles would be restricted in terms of completing downward thrusts. At the same time, however, we calculated that this "alcove" of adaptively-shortened ribs and up-angling of the pelvis would provide an elevated range of motion that would enable the animal to raise its rear flippers higher in the water than the anterior set. (Note: this increased posterior range of motion is supported, in part at least, by reference 5: "Both taxa show a large anteroposterior arc of motion for the hind limb, which may indicate that the hind limbs were important in maneuvering").

Our findings strongly suggest that this anteroposterior arc was not, in fact, primarily for maneuvering, but was, instead, mainly for locomotion. We calculate the resting (neutral) position of the rear paddles was approximately -24 degrees from horizontal (variation amongst different plesiosaur species applies) with dorsoventral ranges estimated at -0 to -37 . These ranges align with previous findings [5, 21], particularly with the former study. When describing the pelvis of Thalassomedon haningtoni (DMNH 1588), Carpenter et al. [5] successfully demonstrated how the ilium overhung the acetabulum. "This overhang is sufficient to prevent the femur from rising above the horizontal. The same restriction is a common feature of plesiosaurs."

With Ava's posterior paddles able to travel higher in the water throughout their positive stroke (with the femurs at or near horizontal), they axially rotate during recovery, then push back hard through the water during the positive stroke, with the trailing edge of the paddles being near-vertical. The posterior paddles end in a far more lateral termination point than the anterior set. In living plesiosaurs this served the purpose of encompassing a different plane of motion than the front flippers, thereby pushing through different water than 
the front pair, and providing both improved acceleration and an overall increase in speed.

\section{A differing range of motion for the anterior paddles}

Unlike the posterior paddles, which moved largely laterally, the anterior paddles engaged in a range of motion more similar to that of extant penguins and sea turtles (but without the latter's ability to achieve extreme dorsal elevation due to the presence of an elbow joint). It is interesting to note that the underwater flight ranges of motion Ava exhibits in her anterior paddles was originally suggested by the Victorian-era paleontologists De La Beche and Conybeare [22], who stated that "the employment and motion of the paddle in swimming has many points of agreement with that of the wing in flight".

We calculate that the resting (neutral) position of the anterior paddles was approximately -30 degrees from horizontal (variation amongst different plesiosaur species again applies) with dorsoventral ranges estimated at +30 to -40 . Our dorsal ranges for the fore-flippers align with previous findings [5] as do our ventral ranges [21]. During the recovery (negative) stroke Ava's fore-flippers exhibited axial rotation that enabled the leading edge to sweep forward and up, cutting the water efficiently as they rose. Unlike the rear paddles, when preparing for a power (positive) stroke the front pair were able to rise up above the midline. From there, they were able to push powerfully down and back, encompassing water separate from that affected by the rear flippers and, with both pairs working in conjunction, giving the animal increased speed and maneuverability.

\section{Flippers working in conjunction (vacuum generation)}

Our findings strongly suggest that, although both pairs of plesiosaur flippers moved simultaneously during the positive/power stroke, during the negative stroke the movements were most effective when they became slightly semi-synchronous. Studies of Ava's movements during her assorted versions supported our earlier hypotheses [23, 24] that the posterior flippers received some benefit from the anterior set. Although the paddles moved through separate planes of motion during the positive (power) stroke, they traveled though similar, close to horizontal ranges during the negative (recovery) stroke. Having the fore-flippers begin their return stroke a split-second before the hind flippers resulted in the leading edge of the fore-flippers pushing water and creating a veritable "vacuum" behind them. This turbulence (akin to the pressure wave dolphins ride ahead of moving ships) would have reduced the friction the posterior paddles experienced during their recovery stroke. In fact, during high-speed "sprints" (such as avoiding a predator or pursuing prey) the power generated by the fore-flippers would have generated enough suction that the posterior pair could virtually "come along for the ride". This allowed the rear paddles to easily catch up to the front paddles in time for a synchronous power (positive) stroke, let them conserve strength and absorb kinetic energy, and allowed for the generation of a more powerful positive stroke.

\section{Inherent flexibility of plesiosaur paddles}

Most previous plesiosaur locomotion studies have assumed that plesiosaurs had rigid or semi-rigid paddles [6]. In fact, the initial versions of plesiosaur swim cycle animations generated for this study [16] shared this assumption. However, our subsequent research, including a hands-on examination/comparison of several living extant sea turtle specimens and an analysis of the rubbery trailing edges of their paddles-tissue that extends far beyond the phalanges-is in accord with suggestions [5] that plesiosaur flippers were inherently flexible. Plesiosaur limbs tend to exhibit considerable hyperphalangy, even more so than extant sea turtles, with the phalanges exhibiting a regular reduction in size to the distal tip of the limb (Figure 4).

In addition, we noted during our examination of plesiosaur flipper fossils (Figure 12) that each phalange is joined to its neighbor by an assortment of ligaments. This plethora of phalanges, along with an abundance of connective tissue, led to both stability and flexibility, along with the ability to feather the limb during parts of the stroke (probably controlled by tendons). This is supported by the previous study [5] which stated: "In plesiosaurs, there is a decrease in the size of phalanges distally, which makes the flipper more flexible distally. This flexibility allows chord-wise flexing with a wave moving distally along the flipper. Many flying and swimming tetrapods can actively control bending and flexing of their wings or flippers with their limb muscles. This manipulation in cetaceans, penguins, sea lions, and sea turtles is mostly by tendons because the distal muscles are reduced or absent [24-27], and this reduction must have been true for plesiosaurs as well."

Although our earliest model had semi-rigid flippers, we found that Ava's flippers performed most effectively during positive (power) strokes when they were endowed with flexibility more in line with that of extant Chelonioidae (as opposed to the more rigid paddles of Spheniscidae). Ava's paddles were adjusted so that they demonstrated a change in shape when under load, becoming "feathered" (backward curving). It should be noted that this type of torsional flexibility is exhibited in sea turtles. During our model's strokes a flexion wave was added that traveled down the limb, producing a powerful flick at the end. Again, this aligns with and confirms the findings of the Carpenter et al. study [5], which refers to the flippers as having a torsional wave (akin to that of sea turtles) that travels down the length of each flipper during the positive stroke and creates additional thrust via a flick at the culmination of each stroke. This was further demonstrated by the use of human swimmers, swimming in tandem and equipped with slightly flexible 
plastic sheets to simulate the paddles of plesiosaurs. As a side note, it bears mentioning that some of the Victorian researchers $[22,28]$ were potentially more accurate in their comparisons of plesiosaurs with extant animals than many "modern" paleontologists. In 1824 Conybeare noted [1] that in "its motion this animal must have resembled the [sea] turtle more than any other."

\section{Effects of up-thrusts}

It should be noted that sea turtles and penguins are able to change direction at will, both dorsally and ventrally, by simply altering the angle of thrust provided by their powerful fore-flippers. Sea turtles, in particular, with their more flexible paddles [14], are able to perform myriad and graceful maneuvers, suspending in place and shifting position in almost any direction they wish, and often by incorporating just one flipper at a time. With plesiosaurs having four well-developed flippers and an extensive system of phalanges/hyperphalanges, we can safely assume that they were capable of doing this as well.

Taking these factors into account, Ava was imbued with one additional effect for added realism; we added an approximation of how the pliosaur's body would respond when exposed to the strain of up-thrusts. When using the fore-flippers (or all four flippers, depending on positioning) to approach the water surface to spout or breathe (a regular occurrence taking place hundreds of times per day) we calculated that the marked increase in water resistance, caused by the sudden shift in stroke angle, would have had a direct effect on the animal's body. The skeletomuscular system would have had to compensate for the sudden and increased resistance during the start of its ascent. We see this skeletomuscular compensation exhibited by both penguins and sea turtles (more apparent in the former, due to the inherent obstruction caused by the latter's shell). Ava was designed to replicate the plesiosaur body's physiological response during its positive (power) strokes by the addition of a stiffening of the spine and a contraction of both the shoulder and abdominal muscles (anchored by robust gastralia). Neck flexion was incorporated to help keep the animal's head/eyes on target, with the head/neck helping to change direction by acting as a rudder.

\section{A breakdown of the strokes}

A full demonstration of our virtual reality pliosaur (including 3D viewing options) is shown below [insert video link here].

In addition, a selection of frames from the animation is available for view (Figure 13). Starting point is top left and moving to the right row by row, typewriter-style (completion of the synchronous stroke takes place in the bottom row, far right).

\section{A discussion on speed}

Although calculations of velocity based on our research have been relegated to a future study, the following notations are provided for consideration. When contemplating the potential speed advantage of a single pair of paddles (as in sea turtles) versus a dual pair (as in plesiosaurs), the most basic analogy would be a comparison of the current world records in sculling-single sculls versus double sculls (currently Robert Manson at 6:30.74 vs Martin and Valent Sinković at 5:59.72). As these statistics demonstrate, the use of dual sets of paddles (rowers) results in a speed increase of only $12 \%$. This is due to the fact that the sculler's oars are traveling through the same water/plane, hence they are subject to the Principal of Flipper Redundancy.

Granted, scullers are not plesiosaurs and, as Carpenter et al. noted [5]: "Despite some estimates of speed for plesiosaurs by Massare [29], we really do not know how fast plesiosaurs routinely swam (cruising speed), nor their burst speed." If we consider, say, the speed of the leatherback sea turtle (Dermochelys coriacea), this largest of extant chelonians has been clocked at a top speed of $35.28 \mathrm{~km} / \mathrm{h}$ (21.92 $\mathrm{mph}$ ), with a typical cruising speed between $1.80-10.08 \mathrm{~km} / \mathrm{h}(1.12-6.26 \mathrm{mph})$ [30]. Given the active piscivorous lifestyle of most warm-blooded plesiosaurs, the speediness and agility of their prey (small fish and squid or, in the case of macrophagous pliosaurs, large fish, sharks, and even other marine reptiles) versus the inertness of the jellyfish that the endothermic leatherback preys upon, and taking into account their need to employ bursts of speed to either obtain prey or avoid becoming it, it is reasonable to assume that their upper-end speeds met or exceeded that of the leatherback. This is further supported by an analysis of the fusiform body-shape of the recently discovered polycotylid plesiosaur Mauriciosaurus, and a comparison between it and the leatherback turtle: "Nonetheless, the long and slim limbs that likely all participated in thrust generation, may have allowed for higher velocities" [31].

\section{Conclusions}

The advanced four-flippered swimming style of plesiosaurs was a great success. Plesiosaurs' unique mode of locomotion supported a variety of forms, from nimble pinniped-like polycotylids to outrageously long-necked elasmosaurs to macropredatory pliosaurs that could exceed extant baleen whales in mass.

Analysis of plesiosaur swim dynamics by means of a digital 3D armature (wireframe "skeleton") of a pliosauromorph ("Ava") demonstrates that: 1, plesiosaurs used all four flippers for primary propulsion; 2, plesiosaurs utilized all four flippers simultaneously; 3, respective pairs of flippers of Plesiosauridae, front and rear, traveled through distinctive, separate planes of motion, and; 4 , the ability to utilize all four paddles simultaneously allowed these largely predatory marine reptiles to achieve a significant increase in acceleration and speed, which, in turn, contributed to their 
dominance in marine habitats for over 160 million years.

\section{Appendix I: Specimens used in this study}

The following real specimens or casts of plesiosaur skeletal remains were used in this study: Cryptoclidus oxoniensis (AMNH 995), Elasmosaurus platyurus (ANSP 10081), Peloneustes philarchus, Cryptoclidus sp. (from the de la Salle Collection), Zarafasaura oceanis (from the Hawthorne Collection). Photographs or renderings of the skeletons of the following genera of plesiosaur were also used: Myerasaurus, Cryptoclidus, Elasmosaurus, Rhomaleosaurus, Plesiosaurus, Archelon, Kronosaurus, Liopleurodon, Trinacromerum, Peloneustes, Thalassomedon, Maresaurus, and Mauriciosaurus. Photographs of the following modern skeletons were used: Zalophus californianus, Dermochelys coriacea, Chelonia mydas, and Spheniscus magellanicus, as was video footage of the following extant animals: Zalophus californianus, Dermochelys coriacea, Chelonia mydas, Pygoscelis adeliae, and Eretmochelys imbricata. Lastly, physical examinations of the paddles and limb girdles of the following live specimens of sea turtles and penguins were performed as part of this study: Lepidochelys kempii, Chelonia mydas, and Aptenodytes patagonicus.

\section{Acknowledgements}

We wish to express our gratitude to computer animator Mathieu Lafreniere, for his unparalleled skill at bringing our virtual pliosaur to life. We thank him not only for his insight and ability, but also his patience and tenacity as we worked hand in hand to recreate a style of underwater flight that hasn't been seen in over 65 million years. Thanks also to Adam Smith for access to the plesiosaur images, Tim Delaney of SeaWorld for a behind-the-scenes tour, Jose Patricio O'Gorman for use of his plesiosaur rib illustration, and Ryosuke Motani for the use of illustrations featured in Plesiosaurs Neo.

\section{Figures}

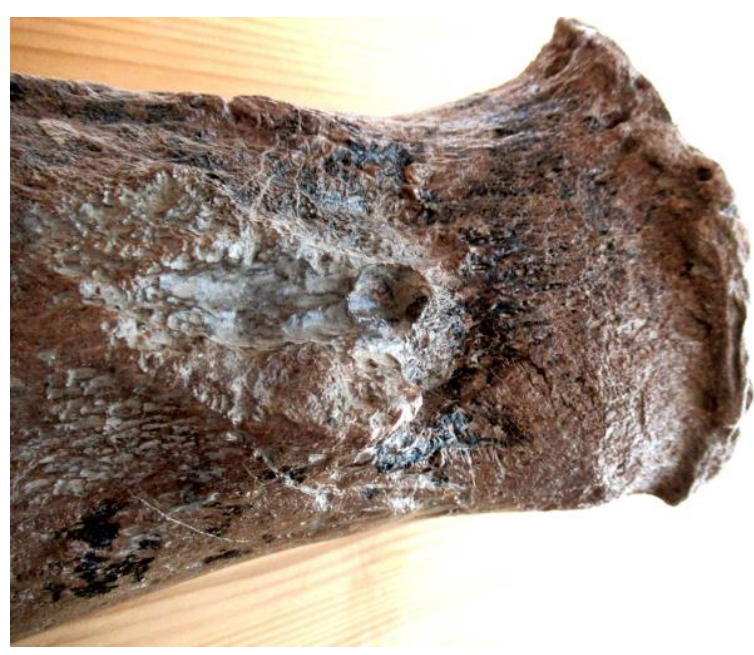

Figure 1. Peloneustes sp. femur, showing muscle attachment site. Specimen from the de la Salle collection.

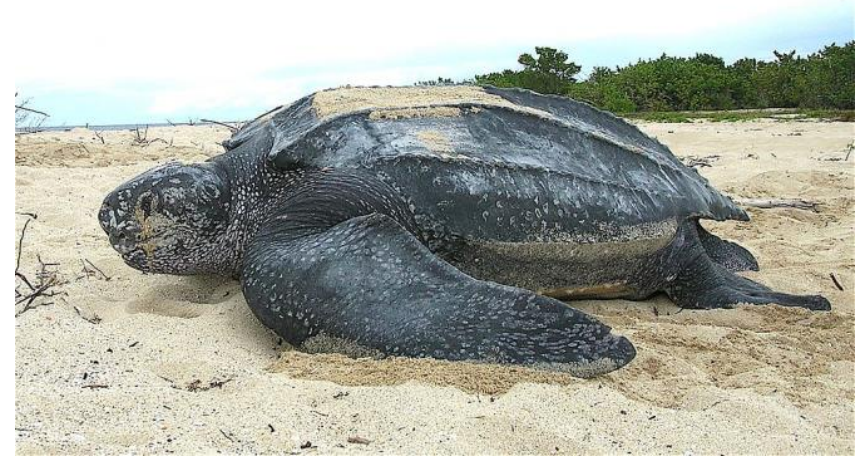

Figure 2. Leatherback Sea Turtle Dermochels coriacea, U. S. Virgin Islands. Public Domain photograph by Claudia Lombard.
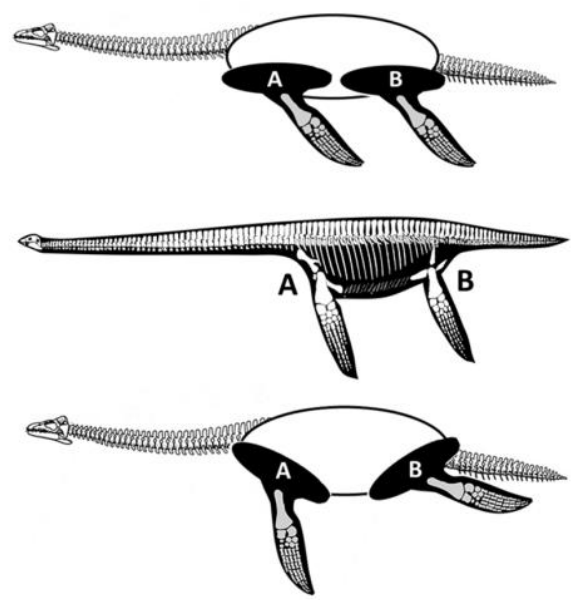

Figure 3. Adjustments to the positioning of front (A) and rear (B) plesiosaur paddles. Adapted from Plesiosaurs Neo by Professor Ryosuke Motani and www.plesiosauria.com (Brown 1981, used with permission). 


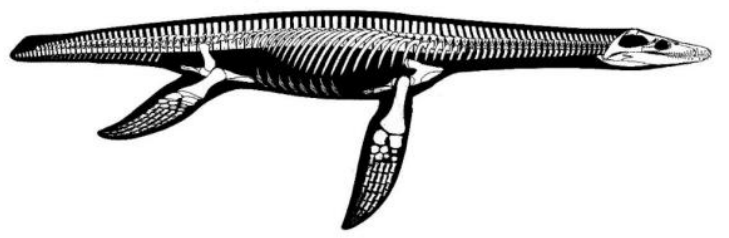

Figure 4. Rhomaleosaurus, based on Smith/Benson 2014 but with adjusted neutral (resting) positioning for the paddles. (used with permission).

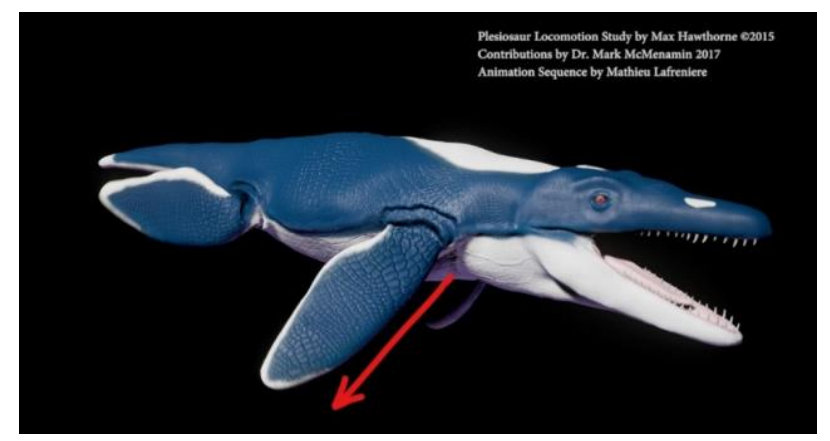

Figure 5. Downstroke of the plesiosaur fore flipper.

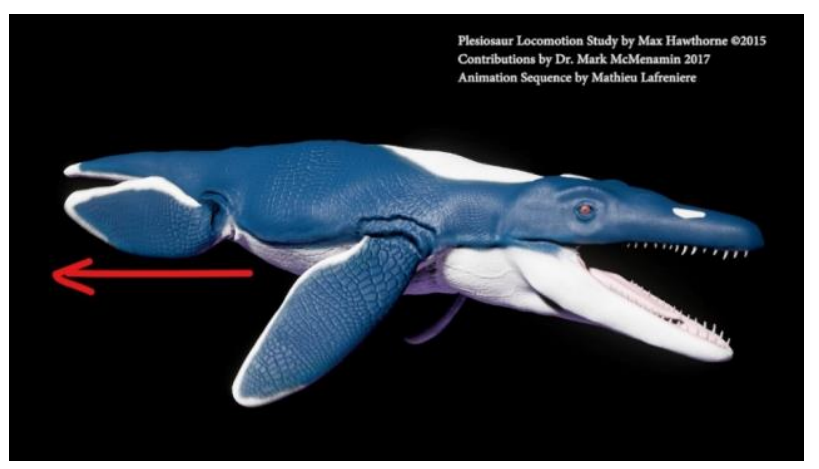

Figure 6. Backward thrust of the plesiosaur hind flipper.

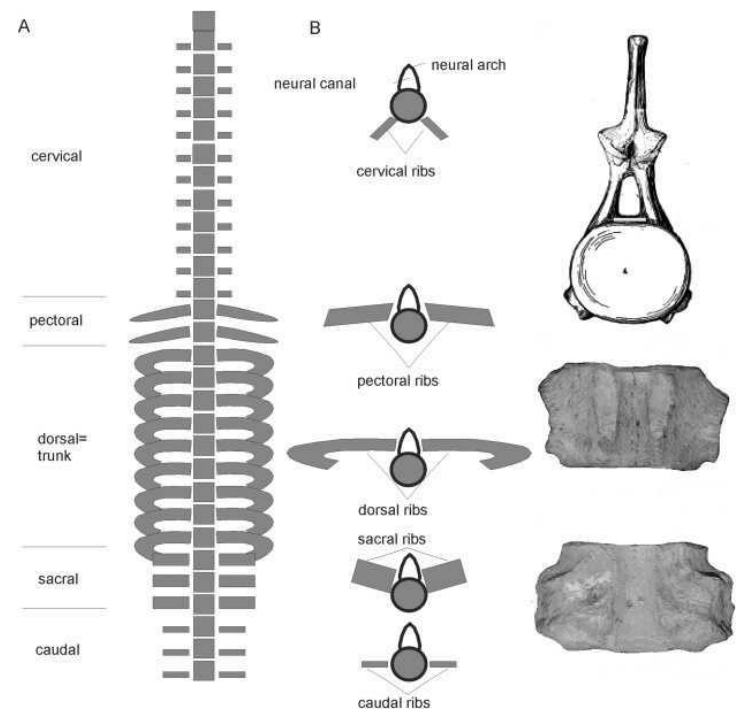

Figure 7. Plesiosaur vertebral column diagram (adapted from O’Gorman/Fernandez 2016, used with permission)

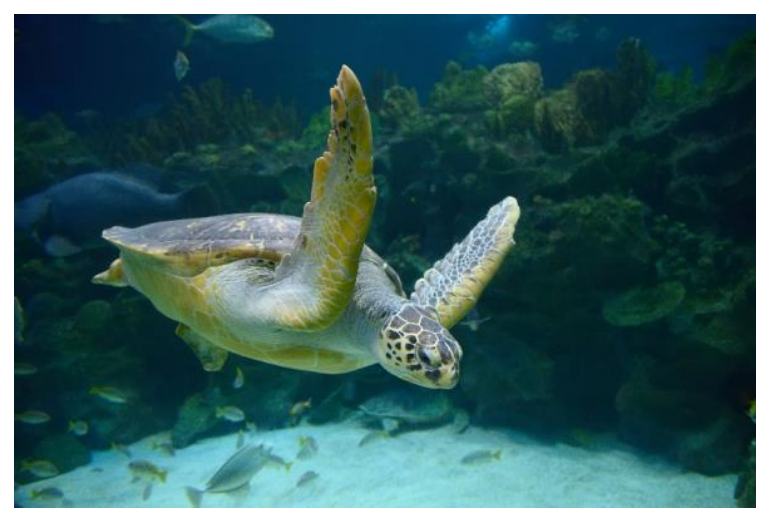

Figure 8. Chelonia mydas, the green sea turtle (licensed image).

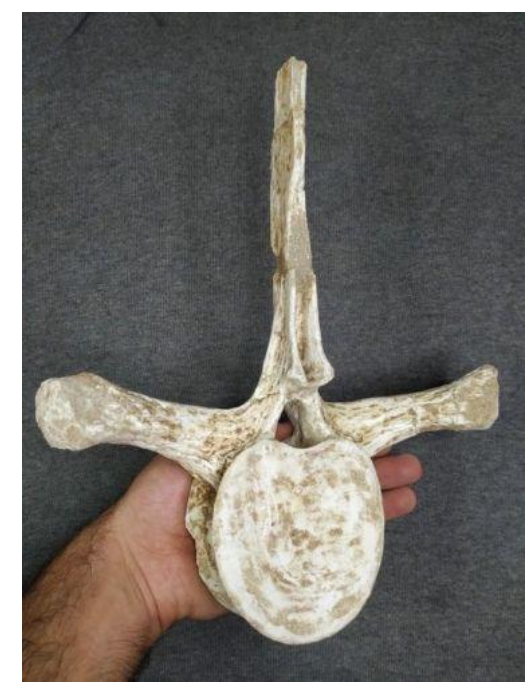

Figure 9. Zarafasaura oceanis sacral (from the Hawthorne collection).

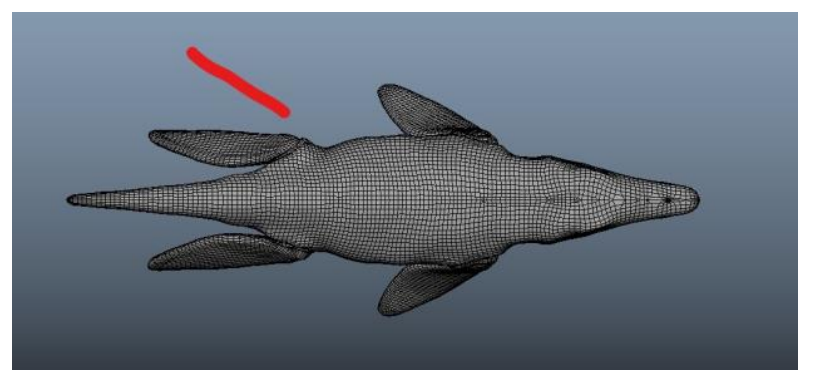

Figure 10. Virtual pliosaur Ava showing contraindicated ranges of motion (red bar) for posterior paddles.

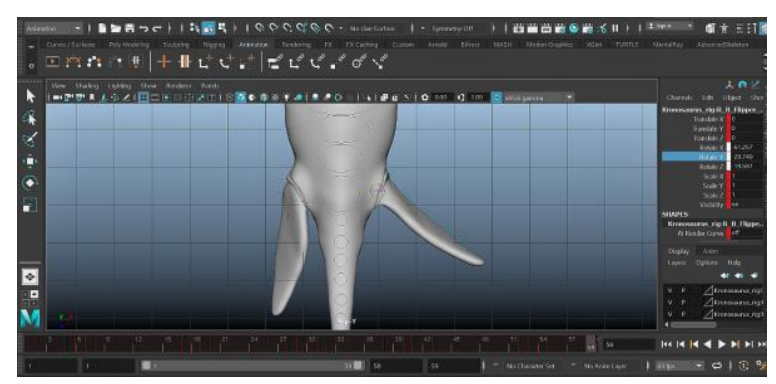

Figure 11. Dorsal view of Ava showing anatomically correct range of motion for right-side posterior paddle. 


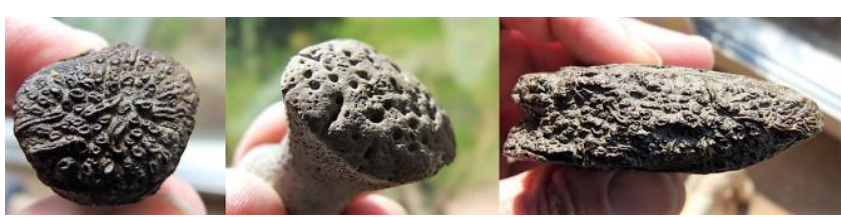

Figure 12. Distal portions of Cryptoclidus phalanges and radius, showing the attachment points of numerous ligaments (from the de la Salle Collection).

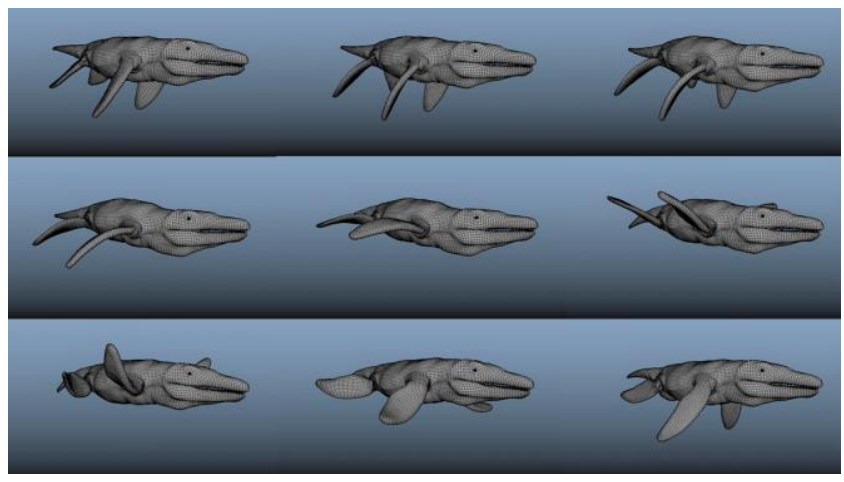

Figure 13. Wireframe swim cycle of Ava (animation by Mathieu Lafreniere).

\section{REFERENCES}

[1] Conybeare, W.D. On the discovery of an almost perfect skeleton of the plesiosaurus. Transactions of the Geological Society of London 1824, S2, 381-389.

[2] Watson, D.M.S. The elasmosaurid shoulder-girdle and fore-limb. Proceedings of the Zoological Society of London 1924, 28, 85-95,.

[3] Robinson, J.A. The locomotion of plesiosaurs. Neues Jahrbuch für Paläontologie, Abhandlugen 1975 149, 286-332,

[4] Robinson, J.A. 1977. Intracorporal force transmission in plesiosaurs. Neues Jahrbuch für Paläontologie, Abhandlugen 1977, 153, 86-128,

[5] Carpenter, K.; Sanders, F.; Reed, B.; Reed, J.; Larson, P. Plesiosaur swimming as interpreted from skeletal analysis and experimental results. Transactions of the Kansas Academy of Science 2010, 113, 1-34.

[6] Muscutt, L.E.; Dyke, G.; Weymouth, G.D.; Naish, D.; Palmer, C.; Ganapathisubramani, B. The four-flipper swimming method of plesiosaurs enabled efficient and effective locomotion. Proceedings of the Royal Society B, Biological Sciences 2017, 284, doi: 10.1098/rspb.2017.0951.

[7] McMenamin, M. Permian Aquatic Reptiles. PaleorXiv 2019, doi:10.31233/osf.io/wb6h7.

[8] Caldwell, M.W. Modified perichondral ossification and the evolution of paddle-like limbs in ichthyosaurs and plesiosaurs. Journal of Vertebrate Paleontology 1997, 17, 534-547.

[9] Taylor, M.A. The lifestyle of plesiosaurs. Nature 1986, 319,
179.

[10] Caldwell, M.W. From fins to limbs to fins: limb evolution in fossil marine reptiles. American Journal of Medical Genetics 2002, 112, 236-249.

[11] Massare, J.A. Swimming capabilities of Mesozoic marine reptiles: a review. In Mechanics and Physiology of Animal Swimming; Maddock, L.; Bone, Q.; Rayner, J.M.V., Eds.; Cambridge University Press, Cambridge, UK, 1994, pp. 133-149.

[12] Rothschild, B.M.; Clark, N.D.; Clark, C.M. Evidence for survival in a Middle Jurassic plesiosaur with a humeral pathology: what can we infer of plesiosaur behavior? Palaeontologia Electronica 2018, 21, 1-11.

[13] Flammang, B.E.; Suvarnaraksha, A.; Markiewicz, J.; Soares, D. Tetrapod-like pelvic girdle in a walking cavefish. Scientific Reports 2016, 6, 23711.

[14] Song, S.H.; Kim, M.S.; Rodrigue, H.; Lee, J.Y.; Shim, J.E.; Kim, M.C.; Chu, W.S.; Ahn, S.H. Turtle mimetic soft robot with two swimming gaits. Bioinspiration and Biomimetics 2016, 11, 036010

[15] Long, J.H.; Schumacher, J.; Livingston, N.; Kemp, M. Four flippers or two? Tetrapodal swimming with an aquatic robot. Bioinspiration \& Biomimetics 2006 1, 20-29.

[16] Buchy, M.C.; Metayer, F.; Frey, E.; Osteology of Manemergus anguirostris $\mathrm{n}$. gen. et sp., a new plesiosaur (Reptilia, Sauropterygia) from the Upper Cretaceous of Morocco. Palaeontographica Abteilung A Palaozoologie Stratigraphie 2005 272, 97-120.

[17] Taylor, G.K.; Nudds, R.L.; Thomas, A.L. Flying and swimming animals cruise at a Strouhal number tuned for high power efficiency. Nature 2003, 425, 707-711.

[18] Sanders, F.; Carpenter, K.; Reed, B.; Reed, J. Plesiosaur swimming reconstructed from skeletal analysis and experimental results. Transactions of the Kansas Academy of Science 2010, 113, 1-34.

[19] Tarlo, L.B. The scapula of Pliosaurus macromerus Phillips. Palaeontology 1958, 1, 193-199.

[20] Godfrey, S.J.. Plesiosaur subaqueous locomotion. Neues Jahrbuch für Geologie und Paläontologie, Monatshefte 1984, 11, 661-672.

[21] Liu, S.; Smith, A.S.; Gu, Y.; Tan, J.; Karen Liu, C.; Turk, G. Computer simulations imply forelimb-dominated underwater flight in plesiosaurs. PLOS Computational Biology 2015, https://doi.org/10.1371/journal.pcbi.1004605.

[22] De la Beche, H.; Conybeare, W.D. Notice of the discovery of a new fossil animal, forming a link between the Ichthyosaurus and crocodile, together with general remarks on the osteology of the Ichthyosaurus. Transactions of the Geological Society of London 1821, 5, 559-594.

[23] Hawthorne, M. Plesiosaur facts: Creatures proper swimming method,

2015 , https://www.kronosrising.com/proper-plesiosaur-swimmingmethods-max-hawthorne/

[24] Hawthorne, M.; McMenamin, M. Plesiosaur swimming method-A flipper propulsion study, 2017, 
https://www.kronosrising.com/plesiosaurs-swam-flipper-pro pulsion-study/

[25] Clark, B.D; Bemis, W. Kinematics of swimming of penguins at the Detroit Zoo. Journal of Zoology, London 1979, 188, 411-428.

[26] Schreiweis, D.O. A comparative study of the appendicular musculature of penguins (Aves: Sphenisciformes). Smithsonian Contributions to Zoology 1982, 341, 1-46.

[27] Fish, F. Structure and mechanics of nonpiscine control surfaces. IEEE Journal of Oceanic Engineering 2004, 29, 605-621.

[28] Seeley, H.G.. Note on some of the generic modifications of the plesiosaurian pectoral arch. Quarterly Journal of the Geological Society 1874, 120, 436-449.

[29] Massare, J.A.. Swimming capabilities of Mesozoic marine reptiles: Implications for method of predation. Paleobiology 1988, 14, 187-205.

[30] Shweky, Rachel, 1999, The Physics Factbook, https://hypertextbook.com/facts/1999/RachelShwe ky.shtml.

[31] Frey, E.; Mulder, E.W.A.; Stinnesbeck, W.; Rivera-sylva, H.E.; Padilla-Gutiérrez, J.M.; González-González, A.H. A new polycotylid plesiosaur with extensive soft tissue preservation from the early Late Cretaceous of northeast Mexico. Boletín de la Sociedad Geológica Mexicana 2017, 69 , http://www.scielo.org.mx/scielo.php?script=sci_arttext\&pid =S1405-33222017000100087\#aff3 